\title{
La humanización en el proceso de formación académica de los profesionales de la salud
}

\author{
Humanization in the process of academic training of \\ health professionals
}

\section{Humanização no processo de formação acadêmica de profissionais de saúde}

Carolina Feliciana Bracarense ${ }^{1}$, Joyce Mara Gabriel Duarte ${ }^{2}$, Helga Marízia Soares ${ }^{3}$, Renata Maciel Côrtes ${ }^{4}$, Ana Lúcia de Assis Simões ${ }^{5}$

${ }^{1}$ Enfermeira. Mestre em Atenção à Saúde pela Universidade Federal do Triângulo Mineiro, Uberaba-MG, Brasil. E-mail: carolinafbracarense@gmail.com

${ }^{2}$ Enfermeira. Doutoranda do Programa de Pós-graduação em Atenção à Saúde da Universidade Federal do Triângulo Mineiro, Uberaba-MG, Brasil.E-mail: joycegduarte@gmail.com

${ }^{3}$ Enfermeira. Mestre em Atenção à Saúde pela Universidade Federal do Triângulo Mineiro, Uberaba-MG, Brasil. E-mail: helguinhamarizia@yahoo.com.br

${ }^{4}$ Enfermeira. Mestre em Atenção à Saúde pela Universidade Federal do Triângulo Mineiro, Uberaba-MG, Brasil. E-mail:renatamcortes@gmail.com

${ }^{5}$ Enfermeira. Doutora em Enfermagem. Professor Associado da Universidade Federal do Triângulo Mineiro. Docente do Programa de Pós-graduação em Atenção à Saúde e Curso de Graduação em Enfermagem, Uberaba-MG, Brasil.

E-mail:ana.assis@reitoria.uftm.edu.br

Cómo citar este artículo en edición digital: Bracarense, C. F; Duarte, J M. G; Soares, H. M; Côrtes, R. M; Simões, A. L. A. (2014) Humanização no processo de formação acadêmica de profissionais de saúde. Cultura de los Cuidados. (Edición digital) 18, 40. Disponible en: <http://dx.doi.org/10.7184/cuid.2014.40.10>

Correspondencia: Rua Coronel Antônio Rios Nº1 Bairro: Mêrces CEP:38061150 Cidade: Uberaba - MG Brasil.

Correo electrónico: carolinafbracarense@gmail.com Recibido: 09/07/2014; Aceptado: 15/09/2014

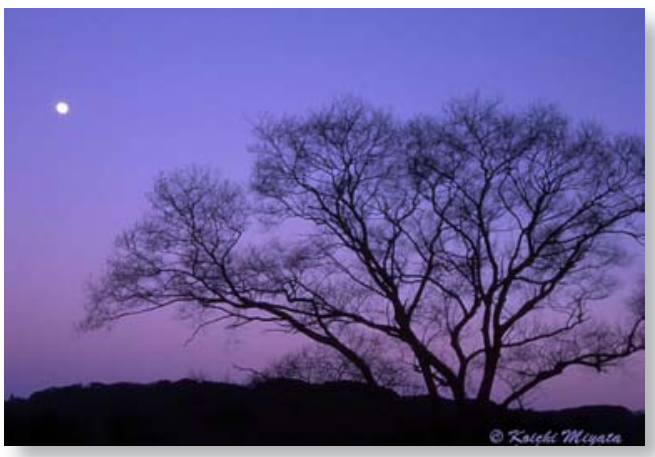

ABSTRACT

Introduction: Observing effective changes in health practices that are compatible with the new model proposed by SUS, offering a reflection on the processes through which health professionals are formed. Researchers point humanization as one of the guiding themes teaching-learning process.
Objective: Identifying, according to the student's opinion, the approach given to the subject of "humanization" during their graduation, and learning their own perceptions upon the subject of "humanization" in healthcare. This is a descriptive, qualitative study. Students participated in the last period of undergraduate courses in Biomedicine, Nursing and Occupational Therapy. The data was collected through the focus group technique, between September and October of 2011. After gathering of the information, the material was submitted to an exploratory reading and, submitted the content analysis of the thematic modality, it was analyzed.

Results and Discussion: Two thematic categories emerged: Humanization according to the perception of the students, and Human- 
ization: transversally crossing between theory and practice. The perception of the students seems to be linked to acts like listening to the user, and being empathetic and resolute. $\mathrm{Hu}-$ manization is a theme that would fundamentally contribute to improve healthcare if approached in professional formation.

Keywords: Healthcare Humanization, Health, Teaching.

\section{RESUMEN}

Introducción: Observar cambios las prácticas de salud que sean efectivos, y compatibles con el nuevo modelo propuesto por el SUS, ofreciendo una nueva reflexión sobre los procesos de formación académica de los profesionales de la salud. Los investigadores señalan la humanización como uno de los temas que orientan la enseñanza-aprendizaje.

Objetivo: identificar, según la opinión de los estudiantes, el abordaje del tema "humanización" en el proceso de formación académica, y conocer la percepción de los estudiantes sobre la humanización en la salud. Es un estudio descriptivo y cualitativo. Los estudiantes participaron del último período de los cursos de Biomedicina, Enfermería y Terapia Ocupacional. Los datos fueron recolectados a través de la técnica de grupo focal, en el período septiembre y octubre 2011. Después, el material fue sometido a la lectura exploratoria y se analizó según la técnica de análisis de contenido de modalidad temática. Dos categorías temáticas emergieron: Humanización en la percepción de los estudiantes, y Humanización: transversalidad entre teoría y práctica. $\mathrm{La}$ percepción de los estudiantes se relaciona a actos como oír el usuario, tener empatía y ser resuelto. El abordaje de la humanización en la formación profesional contribuye fundamentalmente a la mejoría en la asistencia a la salud.
Palabras clave: Humanización de la Asistencia, Salud, Enseñanza

\section{RESUMO}

Vislumbrar mudanças efetivas nas práticas de saúde, que sejam compatíveis com o novo modelo assistencial proposto pelo SUS, proporciona reflexões sobre os processos de formação em saúde. Pesquisadores apontam a humanização como um dos temas norteadores processo ensino-aprendizagem. Este estudo teve como objetivos: Identificar, segundo a opinião dos estudantes, a abordagem do tema humanização durante o processo de formação acadêmica e conhecer a percepção dos estudantes sobre humanização na saúde. Trata-se de estudo descritivo, de cunho qualitativo. Participaram estudantes do último período dos cursos de graduação em Biomedicina, Enfermagem e Terapia Ocupacional. Os dados foram coletados utilizando a técnica do grupo focal, no período de setembro a outubro de 2011. O material foi submetido à técnica de análise de conteúdo na modalidade temática. Emergiram duas categorias temáticas: Humanização na percepção dos estudantes; Humanização: transversalidade entre teoria e prática. A percepção encontrada destes estudantes está atrelada a atos como saber ouvir o usuário, ter empatia e ser resolutivo. A abordagem do tema humanização na formação profissional é fundamental para contribuir na melhoria da assistência à saúde.

Palavras-chave: Humanização da Assistência, Saúde, Ensino.

\section{INTRODUÇÃO}

O sistema de saúde no Brasil, em que pese a situação de constantes avanços, há que se reconhecer que ainda é preciso muito empenho e 
trabalho para sobrepor as dificuldades existentes. Em alusão aos avanços, exemplificam-se as políticas públicas de saúde, as quais instituem programas e projetos que contribuem significativamente para o aumento da qualidade de vida da população. No que tange às dificuldades, ressalta-se a consolidação do modelo de atenção à saúde proposto pelo Sistema Único de Saúde (SUS), que é sustentado pela integralidade da assistência (Souza, Costa, 2010).

Vislumbrar mudanças efetivas nas práticas de saúde, que sejam compatíveis com o novo modelo assistencial proposto pelo SUS, proporciona reflexões sobre os processos de formação em saúde, bem como sobre o perfil profissional que é preciso para atender as novas demandas. Nessa perspectiva, várias ações foram adotadas com vistas a promover inovações educacionais e garantir o respeito aos princípios dos SUS (Hora, Erthal, Souza, Hora, 2013).

Com o intuito de reforçar a necessidade de articulação entre os processos de formação profissional e o modelo assistencial vigente, foi aprovada entre 2001 e 2002 as diretrizes curriculares nacionais de graduação em saúde. Essas diretrizes asseguraram que a formação do profissional de saúde deve contemplar, além do SUS, o trabalho em equipe e a atenção integral à saúde (Almeida, 2003).

Nesse sentindo, ao se considerar o processo de formação dos profissionais de saúde, percebe-se que este não pode ficar restrito aos conhecimentos das áreas técnico-científicas. É preciso que as universidades invistam na formação de cidadãos-trabalhadores da saúde, com capacidade de articular a postura ética, técnica e científica com o respeito a autonomia e subjetividade das relações que se estabelecem nas práticas de saúde (Pasche, 2010; Barbosa, Meneguim, Lima, Moreno, 2013).

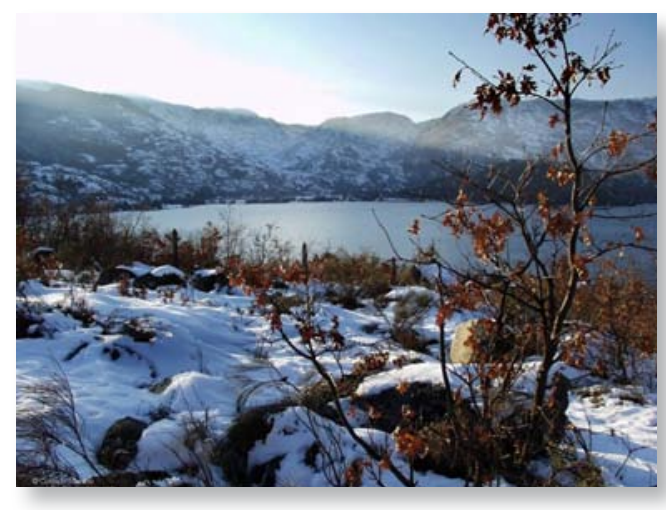

Ao refletirem sobre as transformações vivenciadas pelas sociedades contemporâneas e suas repercussões na formação médica, pesquisadores apontam a humanização como um dos temas norteadores do processo de ensino aprendizagem (Rego, Gomes, Siqueira-Batista, 2008).

Com o intuito de programar estratégias que valorizem os princípios da universalidade, integralidade e equidade do SUS, o Ministério da Saúde elaborou, em 2003, a Política Nacional de Humanização da Atenção e Gestão do SUS (PNH). A humanização, como uma política pública, é regida por princípios norteadores como: transversalidade; indissociabilidade entre atenção e gestão; protagonismo, corresponsabilidade e autonomia dos sujeitos e coletivos (Brasil, 2006).

Todavia, por mais que a $\mathrm{PNH}$ tenha como um de seus eixos a inserção das diretrizes da humanização nas instituições formadoras de profissionais de saúde, observa-se que, na prática, "sua presença no ensino superior ainda é pálida e sôfrega” (Rios, 2009, p. 259).

Nesse contexto, emergiu o seguinte questionamento: como os cursos da área da saúde estão abordando o tema humanização durante a formação acadêmica? Para alcançar respostas a essa interrogação foi realizado esse estudo com os objetivos e identificar, segundo a opinião dos estudantes, a abordagem do tema 
humanização durante o processo de formação acadêmica e conhecer a percepção dos estudantes sobre humanização na saúde.

\section{TRAJETÓRIA METODOLÓGICA}

Trata-se de um estudo descritivo, de cunho qualitativo, que buscou identificar, segundo a opinião dos estudantes, a abordagem do tema humanização no processo de formação acadêmica dos cursos da área da saúde de uma universidade pública federal, do interior de Minas Gerais/Brasil, e conhecer a percepção dos estudantes sobre o significado deste tema.

Foram convidados a participar do estudo, estudantes regularmente matriculados nos cursos de graduação em Biomedicina, Enfermagem, Educação Física, Fisioterapia, Medicina, Nutrição e Terapia Ocupacional da referida instituição. Adotou-se como critério de inclusão estar cursando o último período do curso, e, para a graduação em Medicina, o $9^{\circ}$ período.

Para o acesso aos estudantes, inicialmente foi feito contato com os coordenadores dos respectivos cursos, a fim de informar sobre a pesquisa, esclarecer seus objetivos e solicitar autorização para a coleta dos dados. Nesse momento, solicitava-se, também, que o coordenador viabilizasse uma oportunidade de contato com os estudantes ou que indicasse um professor que pudesse contribuir para esta aproximação.

Apesar de, no primeiro contato, todos os coordenadores apresentarem-se dispostos a contribuir com a pesquisa, somente os cursos de Biomedicina, Enfermagem e Terapia Ocupacional viabilizaram a coleta de dados. Deste modo, a população do estudo foi constituída pelos alunos de biomedicina, enfermagem e terapia ocupacional, cujas turmas totalizavam, respectivamente, cinco, 23 e 17 alunos.

Os dados foram coletados nos meses de setembro e outubro de 2011, utilizando a técnica do Grupo Focal (GF). Acredita-se que a abordagem em grupo incita a discussão entre os participantes e permite que o tema em questão seja mais problematizado. Para realização desta técnica é preciso dispor de uma equipe composta por moderadores e observadores (Dall'agnol, Trench, 1999).

Os grupos focais foram organizados com o apoio dos professores que disponibilizaram um período de tempo durante a disciplina; nos casos em que isso não foi possível, foi feito convite aos estudantes, e agendados horários e salas, de acordo com a disponibilidade da turma. No decorrer dos grupos focais, estiveram presentes: o pesquisador, que fez o papel de moderador, e dois auxiliares, que realizaram as observações e anotações necessárias em um diário. Os estudantes foram identificados com crachás contento números, para auxiliar na posterior transcrição das falas. Ressalta-se que os grupos foram realizados separadamente, de acordo com cada curso, considerando as especificidades existentes. O tempo médio utilizado para a realização de cada GF foi de 50 minutos, sendo necessário um encontro por curso.

$\mathrm{O}$ roteiro foi constituído pelos seguintes tópicos norteadores: entendimento sobre o tema humanização; sua abordagem durante o processo de formação profissional; observação da humanização nas práticas de saúde.

Após a fase de coleta, todas as informações obtidas durante os grupos focais foram transcritas na íntegra, utilizando o computador. O material foi organizado em três conjuntos distintos e submetido à leitura exploratória, buscando uma primeira aproximação com conteúdo das entrevistas. Procedeu-se a análise de acordo com a técnica de análise de conteúdo na modalidade temática, utilizando a adaptação proposta por Gomes (Minayo, 2008). Nesta modalidade direciona-se o caminho 
para as questões subjetivas e permite-se descobertas que não são propriamente evidenciadas pelas palavras, possibilitando apreender o que está além dos conteúdos manifestos pelos indivíduos (Minayo, 2008).

A partir da realização de uma leitura geral dos registros, observou-se que os temas eram comuns a todos os grupos; assim optou-se por fazer uma análise conjunta. Essa leitura geral permitiu a construção da estrutura de análise, possibilitou a identificação dos aspectos centrais e dos núcleos de sentido apontados pelos fragmentos dos relatos. Em seguida, estes foram agrupados em categorias temáticas, que serão apresentados e discutidos à luz da literatura específica.

O projeto desta pesquisa foi aprovado pelo Comitê de Ética e Pesquisa com Seres Humanos da UFTM, com o protocolo número 1853. Para a participação no estudo, cada estudante recebeu, leu e assinou o Termo de Consentimento Livre e Esclarecido.

\section{RESULTADOS E DISCUSSÃO}

Participaram do estudo estudantes dos cursos de graduação em Biomedicina, Enfermagem e Terapia Ocupacional. Os grupos focais foram realizados separadamente por curso e totalizaram a participação de 37 estudantes, sendo quatro de Biomedicina, 17 de Enfermagem e 16 de Terapia Ocupacional.

A partir da análise de conteúdo dos depoimentos, foi possível construir duas categorias temáticas: Humanização na percepção dos estudantes; Humanização: transversalidade entre teoria e prática, as quais serão apresentadas e discutidas a seguir.

\section{Humanização na percepção dos estudantes}

Nesta categoria foram agrupadas as unidades de registro que expressam o significado de humanização na percepção dos estudantes, as quais denotam a compreensão do conceito de humanização, abrangendo atitudes voltadas para os pacientes e, também, para os profissionais de saúde.

Assim, a partir da diversidade na percepção dos estudantes acerca do tema humanização, foi possível reagrupar as unidades de registro em duas subcategorias: Atos de Humanização e Humanização para além do paciente.

\section{Atos de Humanização}

Os estudantes expressaram que a humanização na saúde se manifesta por meio de atos praticados pelos profissionais, como ouvir, respeitar o outro e ser empático. Tal percepção corrobora com a literatura, na qual se defende a ideia de que a humanização assume o significado de colocar-se no lugar do outro e ser respeitoso (Salício, Gaiva, 2011).

$\mathrm{O}$ ato de ouvir, destacado pelos estudantes como prática humanizadora, constitui importante etapa do processo de comunicação; ouvir atentamente e com interesse as pessoas representa uma forma de demonstrar respeito e atenção ao outro.

Estudos apontam que a comunicação deve ser a base do relacionamento entre os profissionais de saúde e usuários. Esta interação permite ao profissional ter visão ampliada do contexto de saúde e doença no qual o usuário está inserido e faz aflorar tanto no usuário quanto em sua família sentimentos de respeito e confiança (Silva, Oliveira, Souza, 2012).

Os depoimentos ilustram a opinião dos estudantes sobre a importância da comunicação no contexto da humanização na saúde. “...prestar uma assistência ao paciente, sabendo ouvir, sabendo como você fala, porque dependendo do tom de voz que você fala com o paciente também, não é humanização [...] ouvir o 
que ele acha [...] trazer ele para o cuidado também... (ENF-17)....isso é uma forma de humanização, você é...levar as informações da saúde com termos populares para que a pessoa saiba o que está acontecendo com ela." (TO-9)

Atitudes de respeito e a necessidade de desenvolver empatia também foram salientadas. Os fragmentos a seguir exemplificam o sentido que os entrevistados atribuem quando agregam a empatia e o respeito à ideia de humanização. “...é você se colocar no lugar da pessoa. Você tratar ela como você gostaria de ser tratado... (ENF- 5)" "Eu acho que um pouco da humanização é olhar para o indivíduo, é pensar nele, se colocar no lugar dele. (TO-3)"

Os depoimentos denotam a percepção dos participantes sobre a importância da empatia para uma assistência humanizada. Ressalta-se que a união entre empatia e respeito norteará as ações para a valorização da subjetividade do usuário, proposta pela Política Nacional de Humanização (Brasil, 2006).

O estabelecimento de vínculo entre os usuários e profissionais é essencial pois consente a co-responsabilização das ações de saúde. Deste modo se pretende reorganizar o processo de trabalho almejando substituir a relação moderada na queixa-conduta para uma relação construída pela troca de experiências. Tais considerações podem ser observados nos depoimentos a seguir (Coelho, Jorge, 2009). "O que eu entendo por humanização seriam aquelas atividades que visam uma aproximação maior do profissional com o paciente... (BIOMED-3)”. “...aumentar a relação das pessoas como seres humanos, não como quem presta o serviço. (BIOMED-3)"

Os relatos obtidos no desenvolvimento dos grupos focais evidenciaram que os futuros profissionais querem ser resolutivos e que percebem a resolutividade como sinônimo de humanização. “...muito mais do que só ser educado com a pessoa, envolve você ser resolutivo com o problema que a pessoa está querendo resolver... (ENF-2)”. “...ser resolutivo... (ENF-5)”

Estudos apontam que a resolutividade é o caminho para se alcançar uma assistência humanizada e completa, e que a integração entre profissionais e usuários favorece a incorporação dessa atitude no ambiente de trabalho (Filho, Souza, Castanheira, 2011).

\section{Humanização para além do paciente}

Prestar uma assistência humanizada requer um olhar holístico para as necessidades do paciente, assim como para tudo que o rodeia. A família precisa ser alvo de ações de cuidado, como educação e promoção de saúde; os profissionais de saúde que prestam o cuidado também devem ter suas necessidades e angústias assistidas. "...humanização não é só humanização com o paciente. Engloba humanização também com os profissionais, com toda a família do paciente... (ENF-5)”.

Foi ressaltada nos depoimentos obtidos com os grupos focais a importância da valorização do universo existente além do paciente, do tratamento e da internação. É indispensável compreender a história de vida, as experiências e respeitar as opiniões de quem recebe o cuidado. A partir dessa compreensão e da soma de conhecimentos busca-se despertar a autonomia do usuário (Pires, Rodrigues, Nascimento, 2010). "Pensar um pouco nesse ser humano, quem que é esse ser humano, qual o conceito desse ser humano, que história de vida ele tem, o que é importante para ele. (TO-3)”. “...[humanização] é você conseguir olhar para aquela pessoa [...] além do paciente [...] você conseguir olhar pra aquela pessoa além do tratamento que ela está precisando, não ficar focado só no vamos tratar, pra ela melhorar e tchau. 
É abordar todos os aspectos [...] o que é importante pra ela. (TO-10)"

Para conseguir uma excelência no atendimento é preciso que os processos de trabalho sejam humanizados, o que significa que o trabalhador de saúde deve ser valorizado com salários dignos e condições de trabalho adequadas (Duarte, 2010). Essa percepção sobre a influência das condições de trabalho na assistência prestada pelos profissionais de saúde foi observada pelos estudantes, ao relatarem suas vivências acadêmicas nos campos de estágio. “...é que às vezes o meio que você trabalha faz com que você preste uma assistência precária, e se a instituição em que você trabalha não tem princípios humanizados, ela não colabora, não te ajuda a agir muitas vezes de forma ética e de forma humanizada, se ela não te dá recursos para uma boa assistência. (ENF-23)”.

A partir dessa visão apresentada pelos estudantes manifesta o cenário de transformações que a saúde está vivenciando. A mudança do modelo biomédico para a atenção biopsicossocial. "[humanização] Um cuidado com o outro, mas não um cuidado no sentido biomédico é um cuidado biopsicossocial... (TO-16)”.

É importante informar que esta subcategoria foi constituída com relatos expressos apenas pelos estudantes dos cursos de Enfermagem e de Terapia Ocupacional. Este fato pode estar relacionado às especificidades dos cursos, bem como aos aspectos filosóficos, teóricos e metodológicos dos Projetos Pedagógicos, que orientam o processo ensino-aprendizagem e direcionam o perfil profissional que se deseja formar.

\section{Humanização: transversalidade entre teoria e prática}

Essa categoria abrange depoimentos referentes à abordagem do tema humanização na formação acadêmica, se este tema foi abordado e a maneira como foi abordado.

Pelos relatos obtidos, os três cursos que participaram do estudo apresentaram o tema humanização durante a formação dos seus profissionais.

Os depoimentos dos entrevistados no grupo focal do curso de Enfermagem apontam que o tema humanização adotou uma postura transversal, perpassando por todas as disciplinas, essa abordagem vai ao encontro do que é preconizado pela $\mathrm{PNH}$, que propõe que a humanização seja uma política transversal (Brasil, 2006). “...mesmo não sendo conteúdo especifico [...] todas s disciplinas de alguma forma abordou [humanização]... (ENF-2)”.

Os diálogos apontam uma correlação entre a teoria e a prática, ressaltando que a construção de laços entre os trabalhadores, estudantes e professores favorece o crescimento pessoal e profissional. "A gente teve na teoria e a partir [...] do estágio, situações no decorrer do estágio, deu pra gente visualizar isso e passar a fazer uma discussão em sala de aula... (ENF4)". "...por mais que tenha sido muito abordado, muito falado, humanização é uma coisa que a gente aprende fazendo na hora. (ENF-3)".

No curso de Terapia Ocupacional, o tema humanização, assim como no curso de graduação em Enfermagem, foi visto de forma transversal. Os recursos didáticos utilizados para explanação do tema humanização foram: aulas expositivas, seminários, casos clínicos, grupos de discussão, entre outros. "A gente aprendeu bastante esses temas, através de teatro, leituras, discussão de casos... era uma aula destinada ao que é encontrada na prática. $\mathrm{Na}$ prática a gente também fazia bastante correlação com a teoria. (TO-2)”.

Os impactos que as atitudes dos professores e dos profissionais que atuam no campo 
exercem no processo de formação, segundo os relatos dos estudantes de Enfermagem e de Terapia Ocupacional. Durante o decorrer dos cursos, estes observaram posturas profissionais variadas, e essas observações contribuíram para o discernimento e o aprendizado da humanização no âmbito profissional.

É preciso focar na ligação entre teoria e prática, o aprendizado abrange a construção de novos laços entre os envolvidos no processo de formação, estudantes, professores e trabalhadores. Essa vivência no campo das atividades práticas favorece o surgimento de novos pensamentos e ideais, contribuindo para a formação de profissionais críticos e reflexivos, capaz de interferir no contexto em que estão inseridos. (Silva, Chernicharo, Ferreira, 2011). “...às vezes nas nossas experiências no estágio, a gente não concorda com certas atitudes de certas pessoas, de certos profissionais que falta ainda o respeito. (ENF-10)”. “...na prática a gente viu a prova exata, o modelo a não ser seguido. Que essa é a nossa realidade prática, a gente viu, o que a gente não deve fazer. [...] é o mais importante, o maior aprendizado que a gente tem, tanto ético quanto de humanização. (TO-3)".

Ainda com as unidades de registro dos cursos de Enfermagem e Terapia Ocupacional foi possível verificar que estes estudantes reconhecem que a construção de uma postura humanizada não é finita. Percebem que são privilegiados por terem em sua formação o tema humanização, e ainda, acreditam que os profissionais com maior tempo de formação e que já estão inseridos no mercado muito tempo, talvez não tenham sido preparados para essas mudanças de postura.

Observou-se que o tema Humanização teve uma singela presença durante a formação do biomédico, sendo abordada em uma aula inserida na disciplina de bioética. Contudo, os estudantes referem experiências extracurriculares com a temática humanização, e estas experiências são vistas como uma possibilidade de aquisição de habilidades práticas pautadas em atitudes humanizadas e que em alguns casos podem vir a não serem desenvolvidas nas atividades propostas na grade curricular dos cursos (Helmo, Simões, 2010). "A gente teve a disciplina de ética... Bioética. E teve uma aula dentro da bioética que falou sobre humanização. (BIOMED-2)". "O que a gente teve de contato com humanização, não posso falar por todos, mas foi na liga onde nós tivemos uma atividade junto com o SARAKURA. Foi uma coisa assim... Digamos extracurricular. (BIOMED-3)".

Afirmam que mesmo sem ter aulas teóricas sobre o tema humanização, o mesmo é observado na prática profissional e os estudantes buscam adotar uma postura humanística. “...em relação à humanização, a gente não fica vendo assim em aula, teorias sobre isso, mas, as condutas são de maneira geral bem humanizada porque na nossa profissão em geral a gente tem que saber ser... Tratar as pessoas de uma maneira humanitária assim... Para ter uma boa relação com o paciente, não pode desprezar esse lado humano de maneira nenhuma... (BIOMED-1)".

Os entrevistados salientam a importância de se adotar uma postura de multiplicadores. Para repassar os conhecimentos, eles exemplificam a utilização de programas de educação em serviço, que constitui um instrumento essencial para instigar os profissionais a adotarem uma postura humanizada. É preciso investir em estratégias didáticas que visem não somente a transmissão vertical de conteúdo e técnicas, mas, que almejem promover a sensibilização do profissional para oferecer um 
cuidado humanizado (Costa, Locks, Klock, 2012). “... a gente está ali [no hospital] pra disseminar essas coisas [humanização] entendeu. O hospital é muito grande, são muitas pessoas, e eu acho que cada um tem uma sementinha para plantar. (TO-16)”. “...os próprios profissionais que não tiveram isso [humanização] durante a formação estar adquirindo esses conhecimentos. Através de educações continuadas. (ENF-24)”.

Essa percepção de que é sempre necessário adquirir novos conhecimentos é importante para o futuro profissional desses estudantes; ademais, a elaboração e implementação de planos de educação permanente para os trabalhadores com base nos princípios da humanização é um dos dispositivos presentes na PNH. (Brasil, 2006).

\section{CONSIDERAÇÕES FINAIS}

Os resultados deste estudo, que buscou a percepção dos estudantes da área da saúde acerca do tema humanização, e também conhecer como este tema é trabalhado durante o processo de formação acadêmica, evidenciaram que os estudantes dos cursos de graduação em Biomedicina, Enfermagem e Terapia ocupacional tiveram contato com tal temática.

A percepção destes estudantes sobre humanização está atrelada a atos que se concretizam em atitudes como saber ouvir o usuário, ter empatia e ser resolutivo. Observou-se também a amplitude que o conceito de humanização adquiriu para os entrevistados, evidenciado pela preocupação dos mesmos em incluir a família no cuidado, ser crítico quanto às condições de trabalho e condutas institucionais.

Com relação à humanização no processo de formação profissional, pode ser identificada uma articulação entre teoria e prática mediante os depoimentos que evidenciavam a importância das atividades práticas para fa-

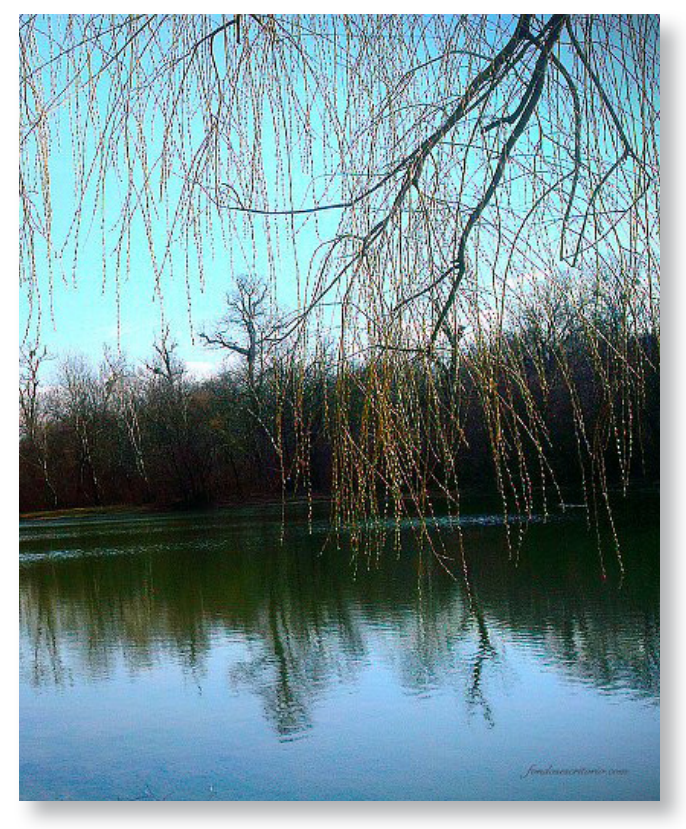

cilitar a assimilação do conteúdo. Os cursos de graduação em Enfermagem e Terapia Ocupacional relataram os exemplos profissionais, e a importância desse compartilhamento de experiências. Os estudantes se intitulam multiplicadores do tema humanização para os profissionais que estão em campo.

Ao identificar no processo de formação acadêmica a abordagem do tema humanização, percebe-se que as universidades que investem nesse tipo de formação podem contribuir para melhoria da assistência à saúde. Haja vista que nos campos de trabalho cada vez mais tem se cobrado a atuação de profissionais comprometidos com a humanização.

Estudos como este contribui para descrever a percepção dos estudantes sobre humanização, e evidenciar a importância da incorporação de atividades práticas nos cursos da área de saúde para possibilitar uma melhor compreensão do processo de formação tanto técnico- científica como reflexiva dos acadêmicos. A limitação deste trabalho foi de ter sido desenvolvido apenas com três cursos da 
área da saúde, fato este que pode estimular o desenvolvimento de novas pesquisas com a intenção de colaborar com o desenvolvimento cientifico da temática.

Assim, os investimentos na formação acadêmica de um estudante através de estratégias que visem à melhoria do ensino trazem como consequência uma assistência à saúde mais qualificada e humanizada.

\section{REFERÊNCIAS}

- Almeida, M. (Org.) (2003) Diretrizes curriculares nacionais para os cursos universitários da área da saúde. Rede Unida, Londrina.

- Barbosa, G. C; Meneguim, S; Lima, S. A.M; Moreno, V. (2013) Política Nacional de Humanização e formação dos profissionais de saúde: Revisão Integrativa. Revista Brasileira de Enfermagem 66(1): 123-127.

- Brasil. (2006) Ministério da Saúde. Secretaria de Atenção à Saúde. Núcleo Técnico da Política Nacional de Humanização. HumanizaSUS: documento base para gestores e trabalha- dores do SUS. 3.ed. Ministério da Saúde, Brasília.

- Coelho, M. O; Jorge, M. S. B.(2009) Tecnologia das relações como dispositivo do atendimento humanizado na atenção básica à saúde na perspectiva do acesso, do acolhimento e do vínculo. Ciência \& Saúde Coletiva 1(14):1523-1531.

- Costa R; Locks, M.O.H; Klock, P. (2012) Acolhimento na unidade neonatal: percepção da equipe de enfermagem. Rev. enferm. UERJ 20(3):349-53.

- Dall'agnol', C. M; Trench, M. H.(1999) Grupos focais como estratégia metodológica em pesquisas na Enfermagem. Rev Gaúcha Enferm. 1(20): 5-25.

- Duarte, M. L. C. Noro, A. (2010) Humanização: uma leitura a partir da compreensão dos profissionais da enfermagem. Rev Gaúcha Enferm. 31(4): 685-692.

- Filho, A. D. D; Souza, M. D. D. O; Castanheira, P. H. D. R.(2011) Acolhimento com classificação de risco: humanização nos serviços de emergência. Revista de Trabalhos Acadêmicos [Online].[acesso em 2011 out 26];2(1).
Disponível em: http://revista.universo.edu.br/index.php ?journal $=1$ reta $2 \&$ page $=$ article $\&$ op $=$ view $\&$ path $\% 5$ B $\% 5$ $\mathrm{D}=311$ \&path\%5B $\% 5 \mathrm{D}=238$.

- Helmo, F. R; Simões, A. L. A.(2010) Liga de Humanização SARAKURA: Contribuição para a formação dos profissionais da saúde. Cienc Cuid Saude 9(1):149-154.

- Hora, D. L; Erthal, R. M. C; Souza, C. T. V; Hora, E. L. (2013) Propostas inovadoras na formação do profissional para o Sistema Único de Saúde. Trab. Educ. Saúde 11(3): 471-486.

- Minayo, M. C. S. (2008) Pesquisa Social: teoria, método e criatividade. Vozes, Petrópolis.

- Pasche, D.F. (2010) Humanizar a formação para humanizar o SUS. In: Ministério da Saúde (BR). Política Nacional de Humanização. Formação e Intervenção. Ministério da Saúde, Brasília (DF) 1: 64-71.

- Pires, V. M. M. M; Rodrigues, V. P, Nascimento, M. A. A. (2010) Sentidos da integralidade do cuidado na saúde da família. Rev. Enferm UERJ 4(18): 622-629.

- Rego S, Gomes AP, Siqueira-Batista R.(2008) Bioética e humanização como temas transversais na formação médica. Rev. bras. educ. med., 4(32), 482-491.

- Rios, I.C. (2009) Humanização: a essência da ação técnica e ética nas práticas de saúde. Rev. bras. educ. med. 33(2): 253-262.

- Salicio, D.M.B; Gaiva, M.A.M.(2011) O significado de humanização da assistência para enfermeiros que atuam em UTI. Rev. Eletr. Enf. 2006 [acesso em 2011 out 24] 8(3): 370-376. Disponível em: http://www.fen.ufg.br// revistaqrevista83/v8n3a08.htm.

- Silva, A. A; Oliveira E. C; Oliveira, S. H. A; Souza, N. R.(2012) A humanização do atendimento e a percepção entre profissionais de enfermagem nos serviços de urgência e emergência dos prontos socorros: revisão de literatura. Ciência et Praxis 5(9):77-84.

- Silva, F. D; Chernicharo, I. M; Ferreira, M.A. (2011) A Humanização na ótica de professores e acadêmicos: estado de arte no conhecimento da enfermagem. Ciência Cuidado e Saúde 10(2):381-388.

- Souza, G. C. A; Costa, I. C. C. (2010) O SUS nos 20 anos: reflexões num contexto de mudanças. Saúde e Sociedade 19(3): 509-517. 